\title{
Effect of overground locomotor training on ventilatory kinetics and rate of perceived exertion in persons with cervical motor-incomplete spinal cord injury
}

\author{
Gino S. Panza $\mathbb{D}^{1,2} \cdot$ Jeffrey E. Herrick ${ }^{3} \cdot$ Lisa M. Chin ${ }^{4} \cdot$ Jared M. Gollie $^{5} \cdot$ John P. Collins ${ }^{6} \cdot$ Dennis G. O'Connell $^{7}$. \\ Andrew A. Guccione (iD ${ }^{8}$
}

Received: 1 July 2019 / Revised: 29 August 2019 / Accepted: 8 September 2019

(c) The Author(s), under exclusive licence to International Spinal Cord Society 2019

\begin{abstract}
Study design Pre-post, pilot study.

Objectives To characterize ventilatory $\left(V_{\mathrm{E}}\right)$ responses to exercise following warm-up walking in individuals with chronic incomplete spinal cord injury (iSCI) during constant work rate (CWR) exercise. Secondarily, to investigate $V_{\mathrm{E}}$ and tidal volume $\left(V_{\mathrm{T}}\right)$ variability, and ratings of perceived exertion (RPE) before and after overground locomotor training (OLT).

Setting Research laboratory.

Methods A 6-min CWR walking bout at preferred pace was used as a warm-up followed by 6 min of rest and a second 6min CWR bout at above preferred walking pace. The second CWR bout was analyzed. Breath-by-breath ventilatory data were examined using a curvilinear least squares fitting procedure with a mono-exponential model. $V_{\mathrm{E}}$ and $V_{\mathrm{T}}$ variability was calculated as the difference between the observed and predicted values and RPE was taken every $2 \mathrm{~min}$.

Results Participants $(n=3, \mathrm{C} 4-\mathrm{C} 5)$ achieved a hyperpneic response to exercise in $V_{\mathrm{E}}$ and $V_{\mathrm{T}}$. OLT resulted in faster ventilatory kinetics and reductions of 24 and $29 \%$ for $V_{\mathrm{E}}$ and $V_{\mathrm{T}}$ variability, respectively. A $30 \%$ reduction in RPE was concurrent with the reductions in ventilatory variability.

Conclusions OLT may improve ventilatory control during CWR in patients with cervical motor-iSCI. These data suggest that in some participants with iSCI, ventilation may influence RPE during walking. Future research should investigate mechanisms of ventilatory variability and its implications in walking performance in patients with iSCI.
\end{abstract}

Gino S. Panza

gpanza@med.wayne.edu

1 John D. Dingell Veterans Affairs Medical Center, Detroit, MI, USA

2 Department of Physiology, Wayne State University School of Medicine, Detroit, MI, USA

3 Department of Exercise Physiology, University of Lynchburg, Lynchburg, VA, USA

4 Rehabilitation Medicine Department, National Institutes of Health, Clinical Center, Bethesda, MD, USA

5 Veterans Affairs Medical Center, Washington, DC, USA

6 KPMG, Vienna, VA, USA

7 Department of Physical Therapy, Hardin-Simmons University, Abilene, TX, USA

8 Department of Rehabilitation Science, George Mason University, Fairfax, VA, USA

\section{Introduction}

Spinal cord injury (SCI) is associated with loss of physical capacity and reduced life expectancy as a result of ventilatory and respiratory dysfunction [1]. Altered ventilatory function, which results in increased work of breathing [2,3] $(\mathrm{Wb})$ and ventilatory muscle fatigability [4], can occur due to changes in thoracic and/or abdominal cavity mechanics [5], ventilatory muscle performance [6-8], and/or altered bronchial control [9]. These impairments diminish pulmonary function with respect to inspiratory and expiratory volume [6], and may influence perceptions of effort [10]. Moreover, these ventilatory constraints may contribute, in part, to the atypical ventilatory response previously published [11].

The typical ventilatory response to constant work rate (CWR) exercise is curvilinear with three phases. An immediate rise in minute ventilation $\left(V_{\mathrm{E}}\right)$ is typically seen within the first breath (phase I), with an exponential 
response occurring within $15 \mathrm{~s}$ (phase II). Thereafter, $V_{\mathrm{E}}$ reaches a steady-state (isocapnic, Phase III) when individuals exercise below the anaerobic threshold [12]. For exercise above the anaerobic threshold, phase III (steadystate) will begin to drift upwards [12]. The ventilatory response to exercise has been shown to be intensity dependent with faster cardiorespiratory responses reported with increased exercise intensity [13]. In addition, previous exercise has been shown to accelerate phase II of the ventilatory response in healthy individuals [14]. In participants with complete SCI, Barstow et al. have reported cardiorespiratory kinetics [15] and improved phase II $V_{\mathrm{E}}$ kinetics following functional electrical stimulation [16]. However, in participants with cervical motor-incomplete spinal cord injury (iSCI), we previously reported a linear ventilatory response to CWR before and after 12 weeks of overground locomotor training (OLT), concurrent with reduced ventilatory variability [11]. We are unaware of any reports characterizing the ventilatory response to exercise in participants with iSCI during unaided walking. Thus, the effects of motor-iSCI on pulmonary $V_{\mathrm{E}}$ and tidal volume $\left(V_{\mathrm{T}}\right)$ during volitional locomotion have not been fully described.

The current pilot study was designed to investigate the ventilatory response following a warm-up bout of exercise and increased exercise intensity (i.e., increased walking speed) during unaided treadmill walking in participants with iSCI before and after OLT. In addition, we aimed to investigate ventilatory kinetics, ventilatory variability, and perceived exertion (RPE) before and after OLT. We hypothesized that the warm-up and increased walking speed would result in a phasic response to exercise. In addition, OLT would result in a reduction in ventilatory variability and RPE.

\section{Methods}

\section{Study design and participants}

This study investigated $V_{\mathrm{E}}$ response before and after OLT using a pre- vs. post-test design. Participants completed a 12-week OLT program developed to elicit mechanical and metabolic adaptations to enhance independent ambulatory function. Inclusion criteria included: 18 years or older; motor iSCI classified as AIS C or D at study entry; at least 12-months after injury; ability to stand and initiate a step with or without an assistive device or trainer; and ability to walk safely on a treadmill. The exclusion criteria included: history of ischemic heart, cardiovascular, pulmonary, or metabolic disease; severe concomitant secondary complications following their injury which included significant orthopedic complications, and spasms/contractures leading to unsafe walking (overground or on the treadmill).

\section{Overground locomotion training}

Details of the OLT program have been published elsewhere [17]. Briefly, training used part-to-whole-practice sequences based on the task-specific movements in the gait cycle. Sessions lasted $90 \mathrm{~min}$ and were conducted twice a week for 12 weeks. Training sessions contained five consecutive training segments: joint mobility; volitional muscle activation; task-isolation; task-integration; and activity rehearsal. Participants used only volitional control and training was conducted without body-weight support, robotic devices, or electrical stimulation. All sessions were conducted by two trained personnel.

\section{Treadmill testing protocol}

Individuals stood quietly for 3-5 min prior to a 6-min walking bout at their individually determined preferred walking speed. This walking bout was followed by $6 \mathrm{~min}$ of rest where participants were offered a seat during the first 4 min of rest but were required to stand for the remaining $2 \mathrm{~min}$ prior to a second walking bout. The second walking bout also lasted $6 \mathrm{~min}$, however each participant self-selected a faster walking speed than the first bout. As such, the intensity of work performed was greater in the second bout. Participants were instructed to use as little upper body support during all walking bouts as was necessary for safety and ability to complete the bout. RPE (0-10) [18] were obtained every 2 min of exercise. Participants repeated the treadmill testing protocol following OLT and the same speeds were used for preferred and above preferred walking speeds.

\section{Instruments}

Height and weight were measured with a standard stadiometer (SECA 213) and scale (Health O Meter 400KL), respectively. Cardiorespiratory variables were collected breath-by-breath with an Ultima CardiO2 gas exchange system (MedGraphics, Saint Paul, MN). Variables included oxygen $\left(\mathrm{O}_{2}\right)$ uptake $\left(\mathrm{VO}_{2}\right)$, carbon dioxide production $\left(\mathrm{VCO}_{2}\right), V_{\mathrm{E}}, V_{\mathrm{T}}$, and endtidal partial pressure of $\mathrm{CO}_{2}\left(\mathrm{P}_{\mathrm{ET}} \mathrm{CO}_{2}\right)$. A 12-lead electrocardiogram (ECG; Mortara M12A, Milwaukee, WI) was monitored throughout exercise. Standard preparation methods were used according to guidelines provided by the Ultima system for a Mason-Likar modification. Flow calibration was completed using a $3000 \mathrm{~mL}$ calibration syringe and gas calibrations were performed through the system's gas auto calibration configuration.

\section{Data analysis}

The data presented was collected as part of a larger study that investigated cardiorespiratory adaptations following 
Table 1 Participant characteristics

\begin{tabular}{|c|c|c|c|c|c|c|c|c|c|}
\hline \multicolumn{10}{|c|}{ Characteristics } \\
\hline Participant & Age & Height & Weight & BMI & $\begin{array}{l}\text { Time } \\
\text { since injury }\end{array}$ & Walking speed & Sex & Injury level & AIS \\
\hline 1 & 18 & 185 & 70 & 20.54 & 22 & 0.72 & M & $\mathrm{C} 4$ & $\mathrm{C}$ \\
\hline 2 & 23 & 183 & 71 & 21.35 & 34 & 0.72 & M & C5 & $\mathrm{C}$ \\
\hline 3 & 35 & 188 & 108 & 30.56 & 61 & 0.45 & M & C5 & $\mathrm{C}$ \\
\hline Mean & 25.33 & 185.33 & 83.27 & 24.15 & 39.00 & 0.63 & & & \\
\hline $\begin{array}{l}\text { Standard } \\
\text { deviation }\end{array}$ & 8.74 & 2.52 & 21.43 & 5.56 & 19.97 & 0.15 & & & \\
\hline
\end{tabular}

Age in years (yrs); Height in centimeters (cm); Weight in kilograms (kgs); Time since injury in months; Walking speed in meters per second

AIS American Spinal Injury Association Impairment Scale BMI body mass index
OLT. Part of the findings were used as pilot data to examine whether increased work rate elicited similar cardiorespiratory responses as seen in people without SCI. Cardiorespiratory data from the warm-up bout has been reported previously [11] and were excluded from analysis in the current study (See "Discussion" section for comparison of results). Therefore, analysis was performed on data from the second, and more intense, bout of walking. All data processing was performed using Origin (Origin 2016, OriginLab Corporation, Northampton, MA) on raw (unaveraged) variables. $V_{\mathrm{E}}$ and $V_{\mathrm{T}}$ during the second walking bout were fit for the entire 6-min bout with a regression function as shown in Eq. (1), where $\mathrm{Y}(t)$ represents $V_{\mathrm{E}}$ or $V_{\mathrm{T}}$ as a function of time, $\mathrm{Y}$ (baseline) is the steady-state $\mathrm{Y}$ of the resting data (based on a 60-s fit), amplitude is the difference between resting data and the end-exercise data at $6 \mathrm{~min}$, time delay (TD) reflects the elapsed time until the exponential fit begins, and $\operatorname{tau}(\tau)$ is the time taken to reach $63 \%$ of the achieved amplitude.

$Y(t)=Y$ Baseline + Amplitude $\left(1-e^{-(t-T D p) / \tau p)}\right)$.

Data points outside of the computed $99 \%$ prediction bands were deemed to be aberrant breaths (e.g., sighing, coughing) and were removed from the analysis. After the regression was recomputed, no additional data points were removed. The criterion for demonstrating a hyperpneic response was met if the equation converged to fit the data. Variability for $V_{\mathrm{E}}$ and $V_{\mathrm{T}}$ were computed by taking the observed data and subtracting it from the best fit regression line obtained from Eq. 1. Thereafter, the absolute value was taken so that an average variability around the predicted value could be calculated (Eq. 2, where $\mathrm{Y}$ represents $V_{\mathrm{E}}$ or $V_{\mathrm{T}}$ ).

$\mid$ Variability $\mid=\left(\operatorname{Observed}\left(V_{\mathrm{E}}\right)-\operatorname{Predicted}\left(V_{\mathrm{E}}\right)\right)$.

$V_{\mathrm{E}}$ and $V_{\mathrm{T}}$ variability were averaged for each 2-min segment of the 6-min bout. Thus, each 2-min average coincided with the $\mathrm{RPE}$ rating.
The estimated $\mathrm{Wb}$ was calculated for each breath using the following equation (Eq. 3) [19] which has been previously used for individuals with SCI [20]. These data were then averaged during the same time periods as $V_{\mathrm{E}}$ and $V_{\mathrm{T}}$ variability.

Estimated $\mathrm{Wb}=-0.430+0.0504\left(V_{\mathrm{E}}\right)+0.00161\left(V_{\mathrm{E}}\right)^{2}$.

Ventilated $\mathrm{CO}_{2}$ and $\mathrm{P}_{\mathrm{ET}} \mathrm{CO}_{2}$ were included in the analyses due to the known moderating effect on $V_{\mathrm{E}}$ in humans [20]. Both variables were averaged during the entire 6-min bout of exercise before and after OLT. In addition, $V_{\mathrm{E}}$ was plotted against $\mathrm{VCO}_{2}$ and the slope coefficient was used to provide an index of ventilatory effectiveness [21].

\section{Statistical analysis}

Descriptive analyses were used to compare baseline (preOLT) with post testing (post-OLT). Data have been rounded to the nearest decimal that allows for the percent change to be seen (See Table 2, $V_{\mathrm{E}}$ Amp/Tau). When group means are presented, the data is accompanied by Cohen's $d$ (effect size $=d$ ). A $d$ of $0.2,0.5$, and 0.8 represent a small, medium, and large effect size, respectively [22].

\section{Results}

Key participant characteristics are found in Table 1. All participants had a cervical injury at C4-5, and diagnosed as motor-incomplete SCI AIS C greater than 1 year post injury. No changes in weight were recorded after OLT. The self-selected walking speed for the warm-up bout was $0.28 \pm 0.05 \mathrm{~m} / \mathrm{s}$ (range: $0.22-0.31 \mathrm{~m} / \mathrm{s}$ ). The self-selected higher intensity exercise bout was $0.63 \pm 0.15 \mathrm{~m} / \mathrm{s}$ (range: 0.45-0.72).

Participant 1 exhibited a resting baseline (resting prior to the second exercise bout) minute ventilation of $16 \mathrm{~L} / \mathrm{min}$ 
pre-OLT and $15 \mathrm{~L} / \mathrm{min}$ post-OLT $(\Delta-9 \%)$. This change in minute ventilation was concurrent with a reduction in tidal volume from 620 to $588 \mathrm{~mL} / \mathrm{breath}(\Delta-5 \%)$. Participant 2 exhibited an increase in minute ventilation at rest from 10 to $11 \mathrm{~L} / \mathrm{min}(\Delta+12 \%)$ with a concurrent reduction in tidal volume from 1021 to $816 \mathrm{~mL} / \mathrm{breath}(\Delta-20 \%)$. Participant 3 exhibited an $8 \%$ increase in resting minute ventilation from 15 to $17 \mathrm{~L} / \mathrm{min}$ after 12 weeks of OLT. The increase in minute ventilation post-OLT was accompanied by a $4 \%$ increase in tidal volume from 726 to $758 \mathrm{~mL} / \mathrm{breath}$. As a group, there was a $2 \%$ increase in minute ventilation and a $9 \%$ decrease in tidal volume. Neither minute ventilation $(d=0.1)$ or tidal volume $(d=0.4)$ during rest demonstrated a large effect size. All exercising data is presented in Fig. 1 and Table 2 and Table 3.

When the data was divided into three 2-min segments for analysis (minutes $0-2,2-4$, and 4-6), reductions in $V_{\mathrm{E}}$ and $V_{\mathrm{T}}$ variability were found at all three timepoints (See Fig. 2 $\mathrm{a}-\mathrm{d}$ ). These reductions were the greatest during minutes $2-4$, the time in which individuals typically transition from phase II to phase III. The largest reduction in RPE was seen during the first $2 \mathrm{~min}$ of exercise $(4.33 \pm 1.09$ vs. $2.33 \pm$ $1.15, \Delta-46.15 \%, d=1.78$ ), however reduction in RPE was also seen at minutes $2-4(5.33 \pm 1.15$ vs. $4.00 \pm 1.0, \Delta$ $-25.00 \%, d=1.24)$ and minutes $4-6(6.00 \pm 1.00$ vs. $4.67 \pm 1.15,-22.22 \%$ change, $d=1.24)$.

\section{Discussion}

There are three primary findings from this study that have not been reported in the literature for this population. First, individuals with a cervical iSCI can achieve a phasic response to volitional unaided walking under the conditions of this study (previous exercise and higher walking intensity), albeit with a high degree of individual variability. Second, the phasic response to exercise improved (i.e., became faster) following OLT. Lastly, the data showed medium to large reductions in $V_{\mathrm{E}}$ variability, $V_{\mathrm{T}}$ variability, estimated $\mathrm{Wb}, \mathrm{VCO}_{2}$ and $\mathrm{P}_{\mathrm{ET}} \mathrm{CO}_{2}$, and RPE following OLT (Table 2, Fig. 2a-d). These results match previous research in healthy individuals which indicated that prior exercise [14] and increased exercise intensity [13, 23] was associated with faster and larger cardiorespiratory responses.

Although the control of ventilation during exercise is still debated [24], it has been suggested that the rapid increase in $V_{\mathrm{E}}$ stems from central mechanisms (e.g., Central Command) $[25,26]$, and that afferents from exercising limbs affects the speed of the ventilatory response with an increase in limb movement frequency resulting in a more rapid rise in $V_{\mathrm{E}}$ [27] as the amplitude of $V_{\mathrm{E}}$ may be dependent on $\mathrm{VO}_{2}$ [23]. However, due to the site of injury and its potential effect on the phrenic nerve, the mechanisms that elicit the phasic ventilatory response to exercise may be altered. During increases in $V_{\mathrm{E}}$ and increased work rates, the diaphragm is recruited first and the accessory ventilatory muscles are thought to be used primarily for controlling cavity pressures [28-30]. However, the accessory muscles are recruited to prevent hypoventilation during times of diaphragmatic fatigue [31, 32]. Considering that persons with an SCI can exhibit altered abdominal wall mechanics [5, 8, 33, 34], altered ventilatory drive [35], and decreases in transdiaphragmatic pressure [36], changes in the diaphragm's mechanical alignment and function may have contributed to our findings.

Exercise intensity is known to influence ventilatory kinetics [13, 22]. Casaburi et al., using a cycle ergometer, performed over 162 exercise tests and reported a clear increase in ventilatory amplitude with increasing work rates, but no change in $\tau(\sim 77 \mathrm{~s})$ between work rates of $25-101 \mathrm{~W}$. At increasing work rates of 127 and $177 \mathrm{~W}, \tau$ also increased to $\sim 85$ and $125 \mathrm{~s}$, which coincided with increases in endexercise lactate [13]. These results demonstrate that ventilatory kinetics are faster during lower intensity work rates and begin to slow as exercise intensity increases. Diamond et al. reported similar ventilatory kinetic values for exercise performed under the anaerobic threshold, regardless of whether exercise was initiated at 0 or $25 \mathrm{~W}$, demonstrating similar ventilatory kinetics within the moderate domain [37]. In patients with complete SCI (AIS A), Barstow et al., reported faster ventilatory kinetics following FES training when compared with the same absolute workload, yet similar values for matched relative workloads [16]. Collectively, these results suggest that exercise intensity may influence ventilatory kinetics. As the workloads (i.e., walking speeds) in this study was self-selected by the participants and not based on performance or capacity, the exercise intensity of the bouts performed are unknown and may have changed with overground training. As such, the ventilatory kinetics as reported here may also be reflecting changes in workload and exercise intensity in these participants.

There is a large degree of variability reported between individuals in this study. For example, all individuals reported a reduction in $V_{\mathrm{E}}$ variability, however participants 2 and 3 showed double and triple the variability, respectively, as compared with participant 1 . This was also observed in $V_{\mathrm{E}} \tau$. However, participant 1 was the only participant to demonstrate a reduction in $V_{\mathrm{T}} \tau$. Considering the injury level and AIS scores are similar between participants. In addition, pre-OLT $V_{\mathrm{E}}$ (average) and RPE were similar in all three participants. One potential explanation could be differences in lower extremity strength. Albeit indirect measures of strength, our participants also completed the Berg Balance Test and the Spinal Cord Independence Measures (SCIM). Participant 3, whom had the highest degree of $V_{\mathrm{E}}$ variability, and slowest ventilatory 
Fig. 1 Ventilatory kinetics before and after OLT.

a-c represent participants 1-3

$(1=\mathrm{a}, 2=\mathrm{b}, 3=\mathrm{c})$ ventilatory

kinetics for both pulmonary

minute ventilation and tidal

volume (BTPS). The left graphs are pre-OLT and the right graphs are Post-OLT. Solid lines represent the kinetic curve for the data collected during that time. The dashed line presented in the Pre-OLT data is the postOLT kinetic curves to provide a visual representation of the changes in the ventilatory kinetics before and after OLT in these patients

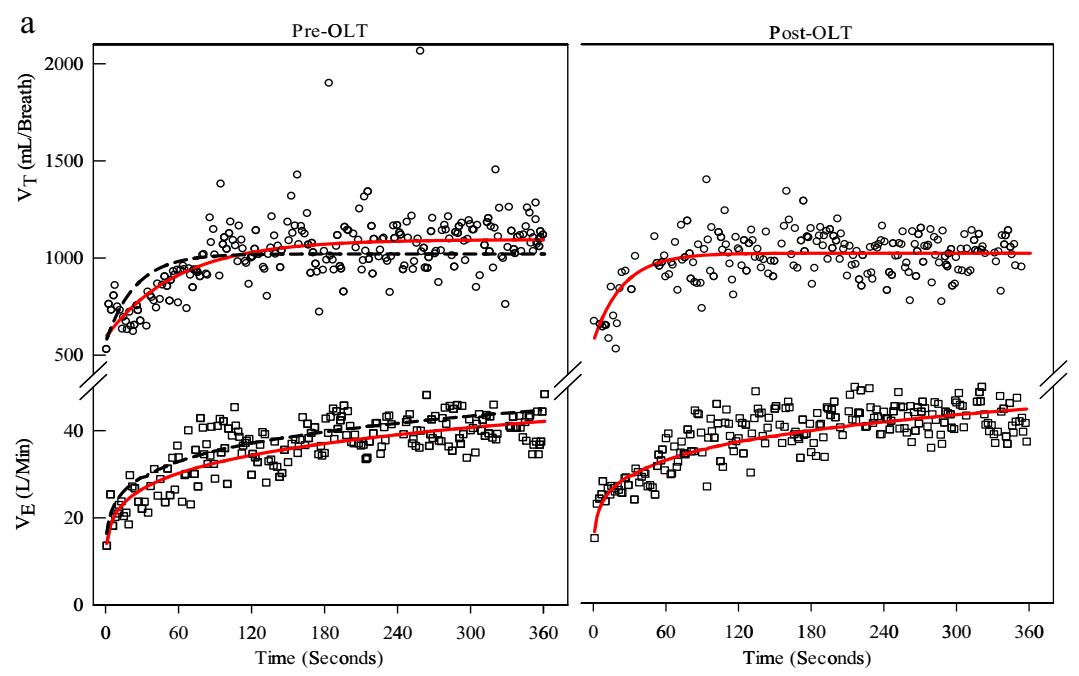

b
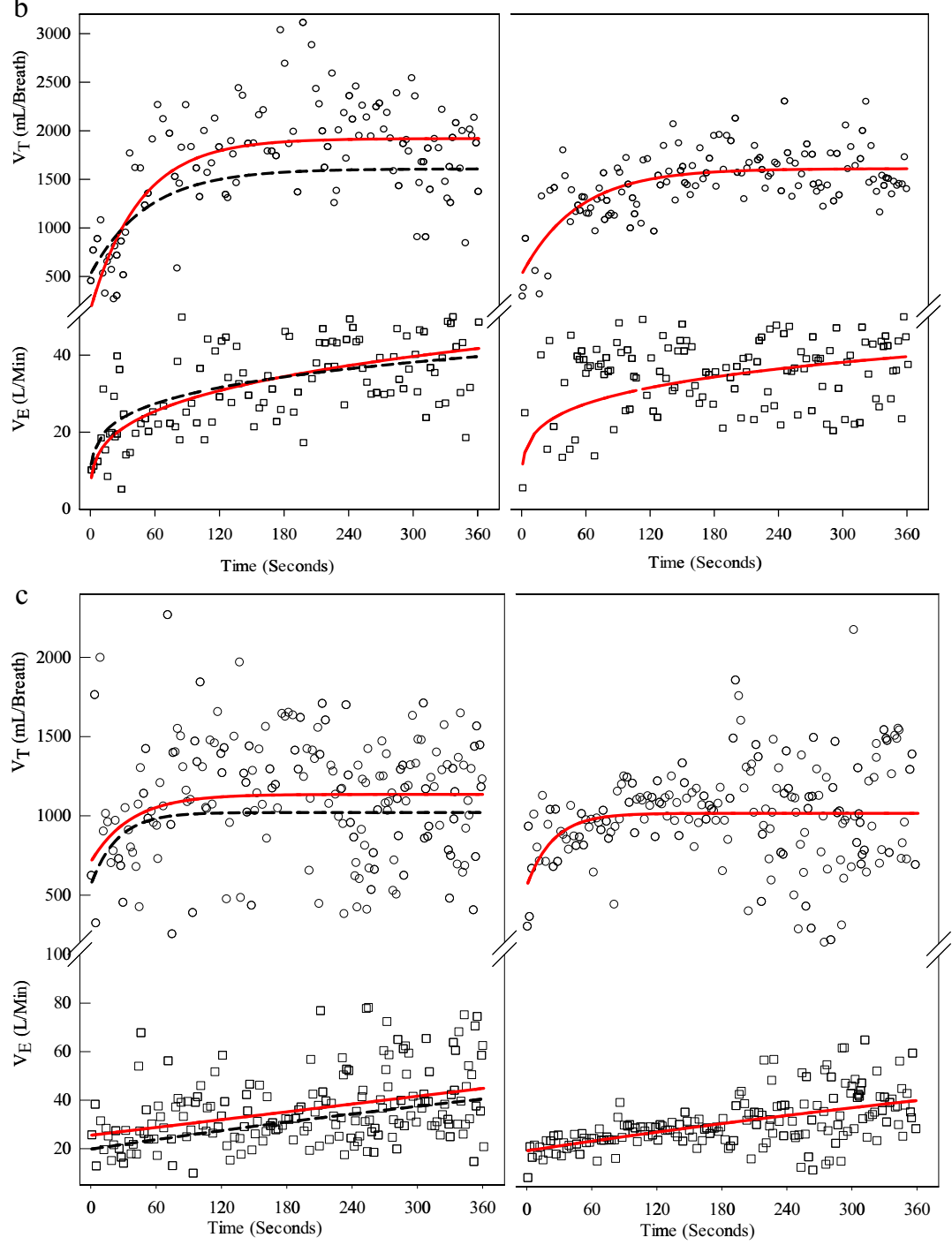

responses (Fig. 1c), may have had the weakest lower body of the three individuals. In the total SCIM participant 1 and 2 scored an 88 compared with participant 3's 78. As for the
Berg total scores, participant 1 and 2 scored a 40 each, and participant 3 scored a 33. In both tests, participant 1 and 2 had marginal improvements and participant 3 had no 
Table 2 Individual changes in averaged 6-min exercise ventilatory variables with group percent change and effect size

\begin{tabular}{|c|c|c|c|c|c|c|c|c|c|c|c|}
\hline & \multicolumn{3}{|c|}{ Participant 1} & \multicolumn{3}{|c|}{ Participant 2} & \multicolumn{3}{|c|}{ Participant 3} & \multirow{2}{*}{$\begin{array}{l}\text { Group } \\
\% \Delta\end{array}$} & \multirow{2}{*}{$\begin{array}{l}\text { Group } \\
\text { Effect size }\end{array}$} \\
\hline & Pre OLT & Post OLT & $\% \Delta$ & Pre OLT & Post OLT & $\% \Delta$ & Pre OLT & Post OLT & $\% \Delta$ & & \\
\hline$V_{\mathrm{E}}(\mathrm{L} / \mathrm{min})$ & 39 & 37 & $-7 \%$ & 34 & 35 & $2 \%$ & 36 & 31 & $-13 \%$ & $-6 \%$ & $0.9 * *$ \\
\hline$V_{\mathrm{T}}(\mathrm{mL} / \mathrm{Br})$ & 1021 & 999 & $-2 \%$ & 1686 & 1475 & $-13 \%$ & 1112 & 1013 & $-9 \%$ & $-8 \%$ & 0.4 \\
\hline Breathing frequency $(\mathrm{Br} / \mathrm{Min})$ & 38 & 37 & $-4 \%$ & 23 & 24 & $5 \%$ & 35 & 34 & $-3 \%$ & $3 \%$ & 0.1 \\
\hline$V_{\mathrm{E}^{-v}}$ ariability $(\mathrm{L} / \mathrm{min})$ & 3.20 & 3.16 & $-1 \%$ & 7.8 & 7.1 & $-9 \%$ & 11 & 6 & $-42 \%$ & $-24 \%$ & $0.6^{*}$ \\
\hline$V_{\mathrm{T}}$-variability $(\mathrm{mL} / \mathrm{Br})$ & 83 & 81 & $-3 \%$ & 333 & 202 & $-39 \%$ & 277 & 205 & $-26 \%$ & $-29 \%$ & $0.7 *$ \\
\hline $\mathrm{Wb}(\mathrm{kg} / \mathrm{m} / \mathrm{min})$ & 4.1 & 3.6 & $-10 \%$ & 3.48 & 3.46 & $-1 \%$ & 4.1 & 3.3 & $-20 \%$ & $-11 \%$ & $1.6^{* *}$ \\
\hline$V_{\mathrm{E}} / \mathrm{VCO}_{2}$ coefficient & 38 & 34 & $-10 \%$ & 48 & 42 & $-14 \%$ & 38 & 35 & $-8 \%$ & $-11 \%$ & $0.9 * *$ \\
\hline $\mathrm{VCO}_{2}(\mathrm{~mL})$ & 1404 & 1245 & $-11 \%$ & 1579 & 1502 & $-5 \%$ & 1248 & 984 & $-21 \%$ & $-11 \%$ & $0.8^{*}$ \\
\hline $\mathrm{P}_{\mathrm{ET}} \mathrm{CO}_{2}(\mathrm{mmHg})$ & 43 & 41 & $-4 \%$ & 52 & 48 & $-8 \%$ & 40 & 35 & $-11 \%$ & $-7 \%$ & $0.5^{*}$ \\
\hline $\mathrm{RPE}(\mathrm{AU})$ & 4.0 & 2.7 & $-33 \%$ & 5.7 & 3.7 & $-35 \%$ & 6.0 & 4.7 & $-22 \%$ & $-30 \%$ & $1.5 * *$ \\
\hline$V_{\mathrm{E}}-\mathrm{Tau}(\mathrm{s})$ & 71 & 68 & $-4 \%$ & 159 & 26 & $-84 \%$ & 140 & 111 & $-20 \%$ & $-44 \%$ & $1.2 * *$ \\
\hline$V_{\mathrm{E}}$-amplitude (L/Min) & 28 & 26 & $-7 \%$ & 37 & 26 & $-30 \%$ & 46 & 39 & $-15 \%$ & $-18 \%$ & $0.8^{*}$ \\
\hline$V_{\mathrm{E}^{-}} \mathrm{Amp} / \mathrm{Tau}(\mathrm{L} / \mathrm{L} / \mathrm{s})$ & 0.39 & 0.38 & $-3 \%$ & 0.2 & 1.0 & $327 \%$ & 0.3 & 0.4 & $6 \%$ & $81 \%$ & $1.2 * *$ \\
\hline$V_{\mathrm{T}^{-}}$Tau $(\mathrm{s})$ & 59 & 24 & $-59 \%$ & 46 & 52 & $14 \%$ & 37 & 41 & $12 \%$ & $-17 \%$ & $0.6^{*}$ \\
\hline$V_{\mathrm{T}^{-}}$amplitude (L/Min) & 475 & 434 & $-9 \%$ & 899 & 792 & $-12 \%$ & 410 & 309 & $-25 \%$ & $-14 \%$ & 0.3 \\
\hline$V_{\mathrm{T}^{-}} \mathrm{Amp} / \mathrm{Tau}(\mathrm{mL} / \mathrm{L} / \mathrm{s})$ & 8 & 18 & $123 \%$ & 20 & 15 & $-23 \%$ & 11 & 8 & $-33 \%$ & $4 \%$ & 0.1 \\
\hline
\end{tabular}

The ventilatory parameters before and after OLT

$L / m i n$ Liters per minute, $\mathrm{mL} / \mathrm{Br}$ milliliters per breath, $\mathrm{Br} / \mathrm{Min}$ breaths per minute, $\mathrm{kg} / \mathrm{m} / \mathrm{min}$ kilograms per meter per minute, $\mathrm{mL}$ milliliter, $A U$ arbitrary units, $s$ seconds

$* d>0.5, * * d>0.8$

Table 3 Correlations

Slope and correlation coefficients

\begin{tabular}{|c|c|c|c|c|c|c|c|c|}
\hline & \multicolumn{4}{|l|}{ All data } & \multicolumn{4}{|c|}{ Participant 2 , post training removed } \\
\hline & $V_{\mathrm{T}^{-}} \mathrm{Tau}$ & $V_{\mathrm{E}}$-variability & $V_{\mathrm{T}}$-variability & RPE & $V_{\mathrm{T}}$-Tau & $V_{\mathrm{E}}$-variability & $V_{\mathrm{T}}$-variability & RPE \\
\hline$V_{\mathrm{E}^{-T a u}}$ & $-0.05(-0.22)$ & $0.03(0.55)$ & $1.43(0.70)$ & $0.02(0.84)^{*}$ & $0.02(0.05)$ & $0.07(0.88)^{*}$ & $2.81(0.99) *$ & $0.03(0.91)^{*}$ \\
\hline$V_{\mathrm{E}}$-variability & & & $30.6(0.87)^{*}$ & $0.36(0.82)^{*}$ & & & $30.95(0.88)^{*}$ & $0.40(0.91)^{*}$ \\
\hline$V_{\mathrm{T}}$-variability & & & & $0.01(0.85)^{*}$ & & & & $0.01(0.91)^{*}$ \\
\hline
\end{tabular}

Slope and correlation coefficients for ventilatory variables and RPE. Slope coefficients (Correlation Coefficients)

$* P \leq 0.05$

change after 12 weeks of OLT. More specifically, the Berg subscores for feet together and ability to pick an object up from the floor were different among all three individuals. In the feet together subtest, participant 1 and 2 scored a 2 and 4 , respectively, as participant 3 scored a 1 . In regards to their ability to pick an object up from the floor, participant 1 and 3 scored a 3 and 4, respectively and participant 3 was unable to complete the task. Based on these findings, participant 1 and 2 may have significantly stronger lower bodies thus improving their motor control during walking and potentially reducing the amount of upper body support used during treadmill walking which could impact their exercising ventilation. Lastly, lower body strength could also have impacted the locomotor-respiratory coupling (discussed below) which could explain the changes in ventilation and ventilatory variability, both among individuals and after OLT, reported here.

Our previous research, which did not include a warm-up exercise bout for the six participants walking at slower speeds, reported the variance of the response via an Fstatistic. When variability was calculated, the group demonstrated a $27.12 \%$ reduction in $V_{\mathrm{E}}$ variability $(3.81 \pm 0.80$ vs. $2.75 \pm 0.99 \mathrm{~L} / \mathrm{min}$ ) [11]. These values were lower in absolute and relative terms than the data reported here. However, comparison of these findings may not be warranted as the participants in our previous study did not achieve a hyperpneic response to training. Thus the regression equation used to calculate variability in the previous study was different than what was used in this study (Linear vs. Eq. 1). $V_{\mathrm{T}}$ data was not analyzed in our previous report. 

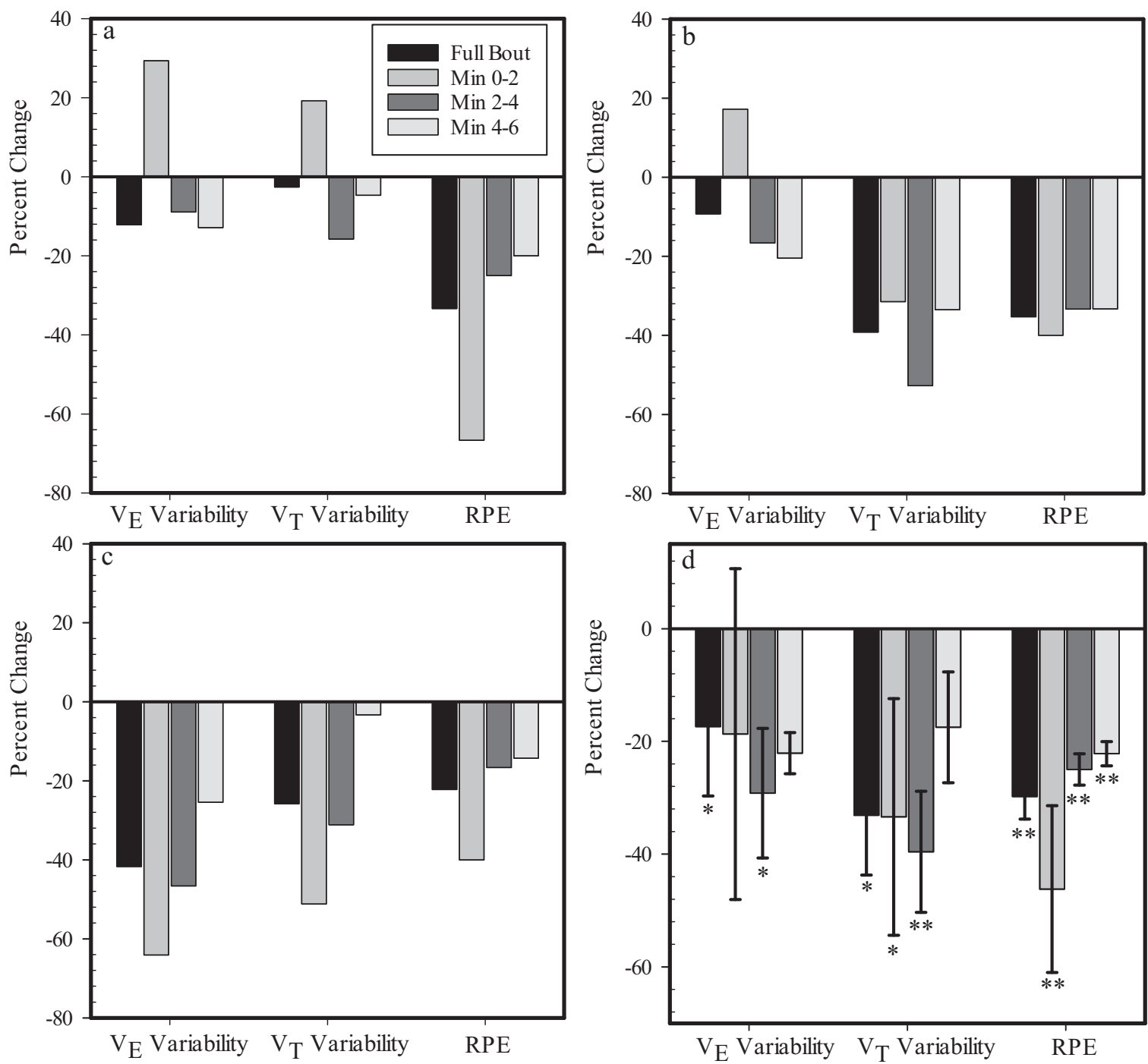

Fig. 2 Individual and group 2 min changes for ventilatory variability and RPE. Graph a-c represent participants $1-3(1=a, 2=b, 3=c)$. Graphs a-c presents the percent change in each $2 \mathrm{~min}$ segment of walking for ventilation, tidal volume, and RPE. In addition, full

There are other potential explanations for the changes in variability reported here. First, typical training adaptations following training may explain our results. For example, increased ventilatory muscle strength may have resulted in improved lung capacity [38, 39] as locomotor training and nonventilatory maneuvers in patients with SCI have been shown to improve pulmonary capacity $[7,40]$ which can increase ventilatory efficiency (e.g., improved ventilation/ perfusion ratio). In addition, due to the changes in walking kinematics following SCI, locomotor-respiratory coupling is another potential explanation for the ventilatory variability reported here. During CWR, ventilation becomes entrained with limb movement which has been attributed, in part, to a "visceral piston" [41], which is the movement of abdominal mass during locomotor activities. If walking

exercise duration averages $(6 \mathrm{~min})$ are also presented as filled dark bars. Graph $\mathbf{d}$ represents the group average change in each variable and is presented as mean \pm standard deviation. Asterisk indicates medium effect size and double asterisks indicate large effect size

synchronicity is lost, then uneven movement of the "visceral piston" could also contribute to the variability being reported here. For example, Participant 3's $V_{\mathrm{E}}$ response to CWR appears linear (Fig. 1c), but has a distinct change in ventilation starting around $160 \mathrm{~s}$ in both $V_{\mathrm{E}}$ and $V_{\mathrm{T}}$ (Fig. 1c) which could be attributable to a loss in walking synchronicity [41]. However, our data cannot parse out these alternative explanations. Thus, future research should explore these possibilities.

We found a large effect on RPE with a reduction of $29.79 \%(d=1.5$, Table 2$)$. As expected, RPE increased linearly as exercise progressed, and was reduced following OLT. However, $V_{\mathrm{E}}$ and $V_{\mathrm{T}}$ did not continue to increase linearly with exercise at either testing timepoint (Fig. 1a-c). Lewis et al. used arm ergometry with three exercise 
intensities to investigate the relationship between $V_{\mathrm{E}}$, heart rate, and $\mathrm{VO}_{2}$ and $\mathrm{RPE}$, and reported a linear increase in all variables with exercise intensity [42], and no difference in RPE between individuals with tetraplegia and paraplegia, which suggests RPE responds to exercise as expected. However, in patients with tetraplegia, RPE was only correlated with heart rate, and not $V_{\mathrm{E}}$ or $\mathrm{VO}_{2}$, during the least intense exercise bout. This is surprising considering RPE is influenced by cardiopulmonary exertion, general exertion, and local muscular fatigue [43]. In this study, $V_{\mathrm{E}}$ tau, $V_{\mathrm{E}}$ and $V_{\mathrm{T}}$ variability all correlated with RPE (Table 3 ). These data suggest that the pulmonary systems ability to respond (Tau) to CWR and maintain effective ventilation may be useful measures to predict exercise endurance during moderate CWR exercise in these participants.

There are several limitations to our study. The first is the small sample size. Conclusions drawn from three participants may not be generalizable. The second limitation is that the participants could use handrails for balance and safety. Therefore, it is unknown how much support was being provided from the upper body during testing. In addition, no measures of pulmonary capacity, blood flow, or muscle activation (e.g., electromyography) were obtained; these may provide explanations on how individuals achieve appropriate ventilation during unaided walking and potential sources for ventilatory variability. A third limitation is that our current methodology cannot parse apart if the phasic response is due to the warm-up bout, or the higher intensity of walking. However, these two parameters were chosen as each have shown to result in faster cardiorespiratory responses, and thus should elicit a phasic response if possible in this population. Lastly, the model fit (Eq. 1) chosen for this study may not be the best model as a systematic test of different fitting parameters and equations was not performed and was beyond the scope of this study. Other models or fitting techniques may provide a better characterization of the ventilatory response in this population, however, we and others have previously published using this model in the SCI population [16, 44, 45].

This study provides preliminary characterization of a ventilatory adaptation following 12 weeks of OLT in participants with iSCI. It appears that at least some participants with iSCI included in this pilot study can achieve a phasic ventilatory response to exercise under certain conditions and that OLT increases the speed of the ventilatory response with concurrent reductions in ventilatory variability, both which coincide with changes in RPE in these subjects.

\section{Data availability}

Data was collected prior to 2018 in which we were not required to enter data into an archive.

\section{Compliance with ethical standards}

Conflict of interest The authors declare that they have no conflict of interest.

Ethical approval All study procedures were approved by the Institutional Review Board at George Mason University (618911). The study conformed to the standards set by the Declaration of Helsinki, except for registration in a public database. All participants provided written consent prior to enrollment in the study.

Publisher's note Springer Nature remains neutral with regard to jurisdictional claims in published maps and institutional affiliations.

\section{References}

1. National Spinal Cord Injury Statistical Center, University of Alabama at Birmingham, 2015 Annual Statistical Report. 2016. https://www.nscisc.uab.edu/PublicDocuments/reports/pdf/2015\% 20NSCISC\%20Annual\%20Statistical\%20Report\%20Complete \% 20Public\%20Version.pdf.

2. McCool FD, McCann DR, Leith DE, Hoppin FG Jr. Pressure-flow effects on endurance of inspiratory muscles. J Appl Physiol. 1986;60:299-303.

3. Manning H, McCool FD, Scharf SM, Garshick E, Brown R. Oxygen cost of resistive-loaded breathing in quadriplegia. J Appl Physiol. 1992;73:825-31.

4. Lin KH, Wu HD, Chang CW, Wang TG, Wang YH. Ventilatory and mouth occlusion pressure responses to hypercapnia in chronic tetraplegia. Arch Phys Med Rehabil. 1998;79:795-9.

5. De Troyer A, Estenne M, Vincken W. Rib cage motion and muscle use in high tetraplegics. Am Rev Respir Dis. 1986;133:1115-9.

6. Schilero GJ, Spungen AM, Bauman WA, Radulovic M, Lesser M. Pulmonary function and spinal cord injury. Respir Physiol Neurobiol. 2009;166:129-41.

7. Mueller G, Perret C, Spengler CM. Optimal intensity for respiratory muscle endurance training in patients with spinal cord injury. J Rehabil Med. 2006;38:381-6.

8. De Troyer A, Heilporn A. Respiratory mechanics in quadriplegia. The respiratory function of the intercostal muscles. Am Rev Respir Dis. 1980;122:591-600.

9. Schilero GJ, Grimm DR, Bauman WA, Lenner R, Lesser M. Assessment of airway caliber and bronchodilator responsiveness in subjects with spinal cord injury. Chest. 2005;127:149-55.

10. Romer LM, Dempsey JA, Lovering A, Eldridge M. Exerciseinduced arterial hypoxemia: consequences for locomotor muscle fatigue. Adv Exp Med Biol. 2006;588:47-55.

11. Panza GS, Guccione AA, Chin LM, Gollie JM, Herrick JE, Collins JP. Effects of overground locomotor training on the ventilatory response to volitional treadmill walking in individuals with incomplete spinal cord injury: a pilot study. Spinal Cord Ser Cases. 2017;3:17011.

12. Wasserman K, Whipp BJ, Casaburi R. Respiratory control during exercise. In: Comprehensive physiology. John Wiley \& Sons, Inc.; 2011. http://onlinelibrary.wiley.com/doi/10.1002/ cphy.cp030217/abstract.

13. Casaburi R, Barstow TJ, Robinson T, Wasserman K. Influence of work rate on ventilatory and gas exchange kinetics. J Appl Physiol. 1989;67:547-55.

14. Ward SA, Whipp BJ. Kinetics of the ventilatory and metabolic responses to moderate-intensity exercise in humans following 
prior exercise-induced metabolic acidaemia. Adv Exp Med Biol. 2010;669:323-6.

15. Barstow TJ, Scremin AM, Mutton DL, Kunkel CF, Cagle TG, Whipp BJ. Gas exchange kinetics during functional electrical stimulation in subjects with spinal cord injury. Med Sci Sports Exerc. 1995;27:1284-91.

16. Barstow TJ, Scremin AM, Mutton DL, Kunkel CF, Cagle TG, Whipp BJ. Changes in gas exchange kinetics with training in patients with spinal cord injury. Med Sci Sports Exerc. 1996;28:1221-8.

17. Gollie JM, Guccione AA, Panza GS, Jo PY, Herrick JE. Effects of overground locomotor training on walking performance in chronic cervical motor-incomplete spinal cord injury: a pilot study. Arch Phys Med Rehabil. 2017;98:1119-25.

18. Robertson RJ, Goss FL, Metz KF. Perception of physical exertion during dynamic exercise: a tribute to Professor Gunnar A. V. Borg. Percept Mot Skills. 1998;86:183-91.

19. Coast JR, Rasmussen SA, Krause KM, O'Kroy JA, Loy RA, Rhodes J. Ventilatory work and oxygen consumption during exercise and hyperventilation. J Appl Physiol. 1993;74:793-8.

20. Foll-de Moro DL, Tordi N, Lonsdorfer E, Lonsdorfer J. Ventilation efficiency and pulmonary function after a wheelchair intervaltraining program in subjects with recent spinal cord injury. Arch Phys Med Rehabil. 2005;86:1582-6.

21. Habedank D, Reindl I, Vietzke G, Bauer U, Sperfeld A, Gläser S, et al. Ventilatory efficiency and exercise tolerance in 101 healthy volunteers. Eur J Appl Physiol. 1998;77:421-6.

22. Cohen J. Statistical power analysis for the behavioral science. 2nd ed. New York, NY: Routledge; 1988.

23. Mateika JH, Duffin J. Changes in ventilation at the start and end of moderate and heavy exercise of short and long duration. Eur $\mathbf{J}$ Appl Physiol Occup Physiol. 1992;65:234-40.

24. Forster HV, Haouzi P, Dempsey JA. Control of breathing during exercise. Compr Physiol. 2012;2:743-77.

25. Eldridge FL, Millhorn DE, Waldrop TG. Exercise hyperpnea and locomotion: parallel activation from the hypothalamus. Science. 1981;211:844-6.

26. Waldrop TG, Eldridge FL, Iwamoto GA, et al. Central neural control of respiration and circulation during exercise. In: Comprehensive physiology. John Wiley \& Sons, Inc.; 2016.

27. Casey K, Duffin J, Kelsey CJ, McAvoy GV. The effect of treadmill speed on ventilation at the start of exercise in man. $\mathrm{J}$ Physiol. 1987;391:13-24.

28. Bellemare F, Grassino A. Effect of pressure and timing of contraction on human diaphragm fatigue. J Appl Physiol. 1982;53:1190-5.

29. Bye PT, Esau SA, Walley KR, Macklem PT, Pardy RL. Ventilatory muscles during exercise in air and oxygen in normal men. $\mathrm{J}$ Appl Physiol. 1984;56:464-71.

30. Romagnoli I, Gigliotti F, Lanini B, Bianchi R, Soldani N, Nerini $\mathrm{M}$, et al. Chest wall kinematics and respiratory muscle coordinated action during hypercapnia in healthy males. Eur J Appl Physiol. 2004;91:525-33.

31. Babcock MA, Johnson BD, Pegelow DF, Suman OE, Griffin D, Dempsey JA. Hypoxic effects on exercise-induced diaphragmatic fatigue in normal healthy humans. J Appl Physiol. 1995;78:82-92.

32. Johnson BD, Babcock MA, Suman OE, Dempsey JA. Exerciseinduced diaphragmatic fatigue in healthy humans. J Physiol. 1993;460:385-405.

33. Estenne M, De Troyer A. Relationship between respiratory muscle electromyogram and rib cage motion in tetraplegia. Am Rev Respir Dis. 1985;132:53-9.

34. Hiraizumi Y, Fujimaki E, Hishida T, Maruyama T, Takeuchi M. Regional lung perfusion and ventilation with radioisotopes in cervical cord-injured patients. Clin Nucl Med. 1986;11:352-7.

35. Golder FJ, Reier PJ, Bolser DC. Altered respiratory motor drive after spinal cord injury: supraspinal and bilateral effects of a unilateral lesion. J Neurosci. 2001;21:8680-9.

36. Hubmayr RD, Litchy WJ, Gay PC, Nelson SB. Transdiaphragmatic twitch pressure. Effects of lung volume and chest wall shape. Am Rev Respir Dis. 1989;139:647-52.

37. Diamond LB, Casaburi R, Wasserman K, Whipp BJ. Kinetics of gas exchange and ventilation in transitions from rest or prior exercise. J Appl Physiol. 1977;43:704-8.

38. Tiftik T, Gökkaya NKO, Malas FÜ, Tunç H, Yalçın S, Ekiz T, et al. Does locomotor training improve pulmonary function in patients with spinal cord injury? Spinal Cord. 2015;53:467-70.

39. DePalo VA, Parker AL, Al-Bilbeisi F, McCool FD. Respiratory muscle strength training with nonrespiratory maneuvers. J Appl Physiol. 2004;96:731-4.

40. Rutchik A, Weissman AR, Almenoff PL, Spungen AM, Bauman WA, Grimm DR. Resistive inspiratory muscle training in subjects with chronic cervical spinal cord injury. Arch Phys Med Rehabil. 1998;79:293-7.

41. Daley MA, Bramble DM, Carrier DR. Impact loading and locomotor-respiratory coordination significantly influence breathing dynamics in running humans. PLoS ONE. 2013;8:e70752-e70752.

42. Lewis JE, Nash MS, Hamm LF, Martins SC, Groah SL. The relationship between perceived exertion and physiologic indicators of stress during graded arm exercise in persons with spinal cord injuries. Arch Phys Med Rehabil. 2007;88:1205-11.

43. Pandolf KB. Influence of local and central factors in dominating rated perceived exertion during physical work. Percept Mot Skills. 1978;46(3 Pt 1):683-98.

44. Gollie JM, Herrick JE, Panza GS, Chin LMK, Guccione AA. Effects of locomotor training on Vo2 on-kinetics in persons with incomplete spinal cord injury: 115 Board \#4 June 1, 9: 30 AM 11: 30 AM. Med Sci Sports Exerc. 2016;48:13-13.

45. Gollie JM, Herrick JE, Keyser RE, Chin LMK, Collins JP, Shields RK, et al. Fatigability, oxygen uptake kinetics and muscle deoxygenation in incomplete spinal cord injury during treadmill walking. Eur J Appl Physiol. 2017;117:1989-2000. 